\title{
SNPs Previously Associated with Dupuytren's Disease Replicated in a North American Cohort
}

\author{
Eric R. Anderson, DO; Zhan Ye, PhD; Michael D. Caldwell, MD, PhD; \\ and James K. Burmester, PhD
}

\begin{abstract}
Objective: Dupuytren's disease is a progressive fibrosis of the hand that often results in debilitating flexion contractures. Its etiology is not completely understood but likely involves both genetic and environmental factors. A recent study performed in Europe identified DNA variants that associate with Dupuytren's disease. Given the likelihood for genetic variation among populations, we planned to validate the genetic variants identified by this study in a North American population.

Methods: In the Marshfield Clinic's Personalized Medicine Research Project, 296 cases with Dupuytren's disease were identified and matched 3-to-I to controls without Dupuytren's disease. Clinical data were abstracted from the electronic medical record. The top 12 single nucleotide polymorphisms (SNPs) from the European study were selected and tested in a multiplex assay using the MassArray Analyzer 4 (Sequenom, Inc., San Diego, CA). Differences in allele frequency were determined, and variants with a $P$ value of $<0.004$ were considered significant.

Results: We replicated 5 of the 12 SNPs previously reported to be associated with Dupuytren's disease.

Conclusion: Our findings support a role for the Wnt signaling pathway in the development of Dupuytren's disease, and suggest that further study of this pathway may result in early diagnosis and non-surgical treatments for Dupuytren's disease.
\end{abstract}

Keywords: Dupuytren's disease; Wnt signaling pathway; Single nucleotide polymorphism;

Fibrotic hand disease; Genetic replication

upuytren's disease (DD) is a progressive fibrosis of the hand that may lead to severe flexure deformities and limit the ability to perform normal activities. It is a common hereditary connective tissue disorder affecting individuals of Northern European descent. ${ }^{1,2}$ Inheritance of DD occurs with variable penetrance. ${ }^{2,3}$ The prevalence of DD ranges from $0.5 \%$ to $11 \%$ in the United States, with the majority of cases over age 60 . Men are affected up to five times more often than women. ${ }^{1-3}$

Correspondending Author: Eric R. Anderson, DO; Department of General Surgery; Divine Savior Health Care; 2817 New Pinery Rd; Portage WI 5390I USA; Tel: 608-742-4I3I; Email: eanderson@dshealthcare.com
At the present time, there is no cure for DD. Treatment options include excision of the diseased fascia, percutaneous fasciotomy, and steroid or collagenase injection. ${ }^{4-6}$ Recurrence of DD ranges from $8 \%$ to $66 \%$, dependent on the severity and treatment of disease. ${ }^{2}$ The high recurrence rate results from failure to treat the underlying cause of $\mathrm{DD}$, which is not completely understood. ${ }^{7}$

Both genetic and environmental factors are likely to contribute to DD. ${ }^{8-10}$ Environmental factors known to affect

Received: September 12, 2013

Ist Revision: December 3, 2013

2nd Revision: December 13, 2013

Accepted: December 18, 2013

doi: $10.3121 / \mathrm{cmr} .2013 .1199$

Funding Support: Marshfield Clinic's Resident Research fund provided the primary financial support for the study. Additional support was provided by grant IUL IRR0250II from the Clinical and Translational Science Award of the National Center for Research Resources, National Institutes of Health. 
the development of DD include repeated hand injuries, diabetes, epilepsy, alcohol consumption, and tobacco use. ${ }^{11-13}$ These factors play a role in inflammation, fibrosis, cellular trauma, and ischemia, affecting the interaction between the myofibroblast and collagen matrix. Altered regulation of growth factor pathways, such as transforming growth factor- $\beta$ and epidermal growth factor, stimulate extracellular matrix formation and myofibroblast activity, increasing the severity of DD. ${ }^{14,15}$

Several studies have investigated the possible genetic causes of DD. One study has shown an association with regions of chromosomes 6,11 , and 16 , with alterations in copy number and structure of chromosomes and possible linkage with DUPC1 at $16 \mathrm{q} .{ }^{8}$ Other studies identified the candidate genes MafB, MMPs, TGF- $\beta$, ADAM 12, POSTN, TNC, ADAMTS3, BMP5, collagenases, $\alpha$-smooth muscle actin, fibronectin, $\beta 1$ integrin, laminin, tenascin C, Hsp47, collagen 1, ALDH1A1, IRX6, PRG4, ZF9, PDGF- $\beta$, and c-myc. ${ }^{15-21}$ Most recently, a whole genome-wide association study performed in Europe, identified eleven single nucleotide polymorphisms (SNPs) from nine different loci, with six of the loci known to be involved in the Wnt signaling pathway. ${ }^{22}$ Because many genetic studies fail replication, and the findings may differ among populations of people, we set out to confirm these findings in a North American population.

\section{Methods}

The study was approved by Marshfield Clinic's Institutional Review Board for human subjects protection. We identified 519 cases of DD in the Marshfield Clinic data warehouse by using International Classification of Diseases Ninth Revision (ICD-9) code 728.6 for palmar fascia contracture. Of those, 296 were found to have genetic data and DNA available in the
Marshfield Clinic Personalized Medicine Research Project (PMRP). ${ }^{23}$ There were 919 age- and gender-matched controls without the diagnosis of DD also identified in PMRP. The PMRP bio-bank contains DNA, plasma, and serum from more than 20,000 subjects residing in central Wisconsin and is linked to the electronic medical record of each subject. ${ }^{23}$ As genetic data are collected, the data are deposited into the genetic repository and made available for approved studies. Clinical data were abstracted for each case and control including race, gender, age at DD diagnosis, hand affected, dominant hand, alcohol use, tobacco use, presence of epilepsy, presence of diabetes, and family history of DD. Eight nonCaucasian subjects were removed at the time of data analysis to prevent spurious associations that could occur from admixture.

\section{Genetic Testing}

Genetic testing was performed using the MassArray Analyzer 4 (Sequenom, Inc., San Diego, CA) and the iPLEX assay that uses primer extension reaction chemistry designed to detect sequence differences at the single nucleotide level. DNA samples were amplified in a multiplexed reaction and extended with a primer specific for the target SNP sequence. The top 12 SNPs from the Dolmans et $\mathrm{al}^{22}$ study were selected for this replication study. Matrix-assisted laser desorption/ ionization time-of-flight (MALDI-TOF) mass spectrometry was used to detect the difference in mass that differentiates SNP alleles. Twenty-three samples from the HapMap-CEU CEPH DNA collection (Coriell Institute, Camden, NJ) were tested as positive controls. These HapMap samples were chosen to represent examples of heterozygous and each homozygous genotype. All genotyping results were compared to the Genotype and Allele Frequency Report from NCBI dbSNP when available for the 12 SNPs included in the

Table 1. The 12 SNPs tested in this association study using 296 cases and 919 controls.

\begin{tabular}{|c|c|c|c|c|}
\hline \multirow[b]{2}{*}{ Gene } & \multirow[b]{2}{*}{ Chromosome } & \multirow[b]{2}{*}{ SNP \# } & \multicolumn{2}{|c|}{$P$ value } \\
\hline & & & Anderson & Dolmans \\
\hline \multirow[t]{2}{*}{ Wnt7Ba } & 22 & rs8140558 & $4.104 \times 10^{-5}$ & $1.2 \mathrm{E}-22$ \\
\hline & & rs6519955 & $1.3 \times 10^{-4}$ & $3.2 E-33$ \\
\hline \multirow[t]{2}{*}{ C8orf34,SULF1a } & 8 & rs1365415 & $2.096 \times 10^{-4}$ & 2.8E-07 \\
\hline & & rs2912522 & $5.361 \times 10^{-2}$ & $2.0 \mathrm{E}-13$ \\
\hline EPDR1,SFRP4 $4^{a}$ & 7 & rs16879765 & $1.336 \times 10^{-3}$ & 5.6E-39 \\
\hline DUXA, ZNF264 & 19 & rs11672517 & $3.058 \times 10^{-3}$ & $6.8 \mathrm{E}-14$ \\
\hline EIF3E,RSPO2 & 8 & rs611744 & $2.348 \times 10^{-1}$ & $7.9 \mathrm{E}-15$ \\
\hline MAFB & 20 & rs8124695 & $9.156 \times 10^{-1}$ & $7.6 \mathrm{E}-10$ \\
\hline DMRT2 & 9 & rs10809650 & $4.930 \times 10^{-1}$ & $6.2 \mathrm{E}-09$ \\
\hline WNT2 & 7 & rs4730775 & $5.834 \times 10^{-1}$ & 3.0E-08 \\
\hline DMRT2 & 9 & rs10809642 & $9.866 \times 10^{-1}$ & $1.2 \mathrm{E}-08$ \\
\hline TIMP2 & 17 & rs4789939 & $9.247 \times 10^{-1}$ & 6.0E-07 \\
\hline
\end{tabular}

aSNPs with a $P$ value less than 0.004 and are considered replicated. 


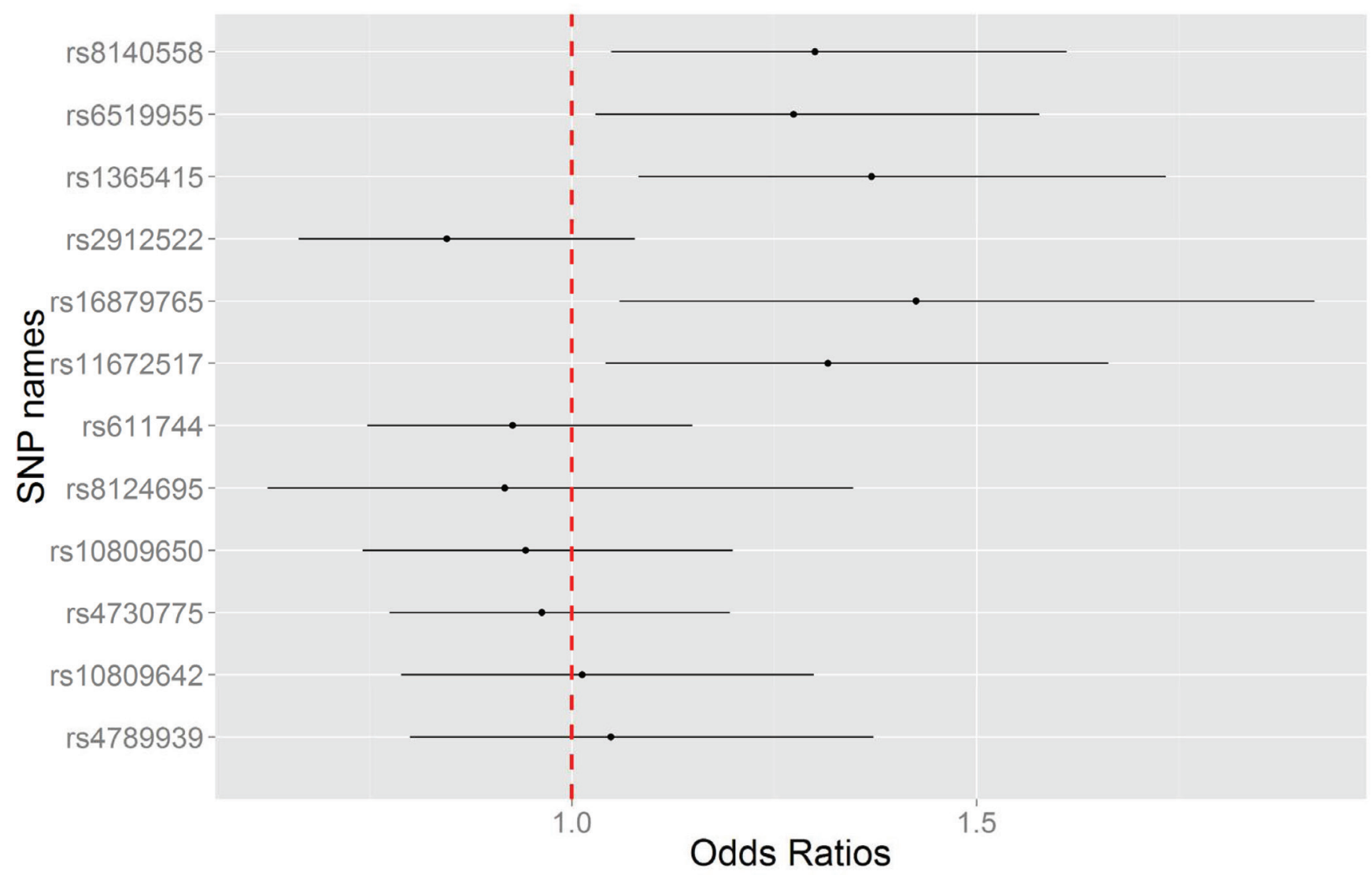

Figure 1. Forest plot showing the odds ratios for the 12 SNPs tested. The five replicated SNPs have higher odds ratios with confidence intervals above 1.0.

Sequenom assay. In addition to the study samples, each 384well plate also included six of the HapMap samples with two no-template controls that were compared to the expected genotypes for quality control.

\section{Statistical Analysis}

Quality assurance was performed on the genetic data prior to statistical analysis. This included ensuring data were available for each SNP on at least $90 \%$ of the subjects and that genotypes were within Hardy-Weinberg equilibrium set at $10^{-3}$. Differences in allele frequency were determined, and the $P$ values from logistic model and Fisher exact tests were reported. The SNPs with a $P$ value $<0.004$ were considered replicated to account for multiple testing (Bonferroni correction). We incorporated the genetic models (additive, dominant and recessive), since we do not know the mode of inheritance of Dupuytren disease. All the procedures and calculations were done using the PLINK software. ${ }^{24}$ In addition, we calculated the odds ratios estimates from each of the markers with the confidence intervals.

\section{Results}

The PMRP population with DD was made up of $62 \%$ men and $38 \%$ women, of which $98 \%$ were white non-Hispanic, $34 \%$ were diabetic, $18 \%$ reported regular use of alcohol, $29 \%$ reported tobacco use, and $4 \%$ had epilepsy. The distribution of white non-Hispanic subjects in PMRP is Germany (74.4\%),
Poland/Czech Republic (7\%), Sweden/Norway (5.8\%) and British and Irish Isles (12.8\%). ${ }^{25}$ Approximately 6\% of our cases had a family history of DD which is much less than the $18 \%$ previously reported in one study ${ }^{1}$ and approximately $40 \%$ in another study. ${ }^{8}$ It is possible that our rate is an underestimate do to inability to capture a complete family history through the electronic medical record where some family members may not be diagnosed or treated and a complete family history may not have been collected by the medical staff.

Of the 12 SNPs tested, we were able to replicate 5. Table 1 shows the $P$ values for the 12 SNPs in both our cohort and that of Dolmans et al (the meta-analysis $P$ values). ${ }^{22}$ It highlights the 5 SNPs with $P$ values $<0.004$ that were considered replicated.

Figure 1 shows the forest plot generated to show the final results for the 12 selected SNPs. The odds ratio reports the ratio between two proportions, which, in the context of our study, are the proportion of individuals in the DD group having a specific allele and the proportions of individuals in the matched control group having the same allele. An odds ratio higher than 1 indicates that the allele frequency in the DD group is much higher than in the control group, that also shows the tested SNPs are associated with the condition. The confidence interval estimation illustrates the precision of the 
estimation of the odds ratio. In our study, we showed that all five identified significant SNPs have higher odds ratios $(>1)$, and also have confident intervals above 1, showing the estimated odds ratios were precisely calculated.

\section{Discussion}

Dupuytren's disease can be difficult to diagnose, and patients may present with advanced disease, making non-operative therapy difficult and ineffective. Additionally, with operative therapy associated with risk of neurovascular injury and recurrence rates between $8 \%$ to $66 \%,{ }^{2}$ early detection is critical. The inability of current treatments to target the unknown, underlying pathology of the disease underscores the importance of identifying an etiology that may allow for earlier detection and better treatments.

The discovery and replication of a genetic cause for DD should allow for long-term development of disease prediction models before the onset of any symptoms, allowing individuals at higher risk of DD to have the option of modifying their activities and limiting exposure to environmental factors associated with DD. Also, understanding the molecular pathways that predispose individuals to DD should lead to a better understanding of the disease pathophysiology, allowing for development of novel targeted interventions.

Although it is well-established that fibrosis is important in DD, little is known about the underlying mechanisms that lead to the fibrotic changes seen in DD. It has been hypothesized that a combination of genetic and environmental interactions results in the disease. Therefore, a single genomewide search for underlying genetic variants that may predispose individuals to DD was performed by Dolmans et $\mathrm{al}^{22}$ in a European population. It identified eleven SNPs from nine different loci, with six of the loci known to be involved in the Wnt signaling pathway. However, many genetic association studies cannot be replicated due to artifacts in the design of the original study, and replication in multiple cohorts often fails to show a true association with disease. We were able to replicate the findings of Dolmans et $\mathrm{al}^{22}$ in a North American population, with the identification of five SNPs with significant $P$ values. However, our cohort was relatively small and some of the tested SNPs may not have reached statistical significance due to our small sample size. Additional studies in larger cohorts will be necessary to investigate interactions between the SNPs and to understand how the individual SNPs contribute to disease.

Of the nine loci identified in the Dolmans et al study, ${ }^{22}$ six are involved in the Wnt signaling pathway, suggesting that misregulation of this pathway contributes to fibrosis seen in DD. Within this pathway, $\beta$-catenin is a key signaling molecule regulated by Wnt whose expression is dysregulated in DD. ${ }^{26,27}$ Currently, it is unknown how the SNPs affect gene expression within the Wnt pathway. One study demonstrated down-regulation of Wnt11 in surgically removed tissue, ${ }^{28}$ and another study demonstrated an increase in $\mathrm{Wnt} 5 \mathrm{a},{ }^{26}$ with a third study demonstrating that the microRNA profile in DD is altered, including that for $\mathrm{Wnt5a}{ }^{29}$ The Wnt family of proteins regulates cell growth, proliferation and differentiation and has been linked to abnormal proliferation of fibroblasts, and appears necessary for TGF- $\beta$-mediated fibrosis seen in fibrotic diseases. ${ }^{30,31,32}$ Modulation of Wnt signaling ultimately leads to changes in gene expression that impact cell proliferation and survival.

Several additional studies implicated the Wnt pathway in DD, although they did not identify specific SNPs that predispose to genetic disease. ${ }^{33,34}$ Our study supports the findings of the Dolmans et al study. ${ }^{22}$ Larger consortium studies can utilize these results to develop risk stratification and, potentially, novel detection methods and treatments for DD.

\section{Acknowledgements}

The authors thank Lynn Ivacic of the Marshfield Clinic Research Foundation's Integrated Research and Development Laboratory for performing the genetic testing. The authors also thank the Marshfield Clinic Research Foundation's Office of Scientific Writing and Publication for assistance in preparing this manuscript.

\section{References}

1. DiBenedetti DB, Nguyen D, Zografos L, Ziemiecki R, Zhou X. Prevalence, incidence, and treatments of Dupuytren's disease in the United States: results from a population-based study. Hand (N Y) 2011;6:149-158.

2. Worrell M. Dupuytren's disease. Orthopedics 2012;35:52-60.

3. Black EM, Blazar PE. Dupuytren disease: an evolving understanding of an age-old disease. J Am Acad Orthop Surg 2011;19:746-757.

4. Desai SS, Hentz VR. The treatment of Dupuytren disease. J Hand Surg 2011:36:936-942.

5. Hentz VR, Watt AJ, Desai SS, Curtin C. Advances in the management of Dupuytren disease collagenase. Hand Clin 2012; 28:551-563.

6. Chen NC, Srinivasan RC, Shauver MJ, Chung KC. A systematic review of outcomes of fasciotomy, aponeurotomy and collagenase treatments for Dupuytren's contracture. Hand (N Y) 2011;6:250-255.

7. Picardo NE, Khan WS. Advances in the understanding of the etiology of Dupuytren's disease. Surgeon 2012;10:151-158.

8. Michou L, Lermusiaux JL, Teyssedou JP, Bardin T, Beaudreuil J, Petit-Teixeira E. Genetics of Dupuytren's disease. Joint Bone Spine 2012:79:7-12.

9. Burge P. Genetics of Dupuytren's disease. Hand Clin 1999; 15:63-71.

10. Capstick R. Bragg T, Giele H, Furniss D. Sibling recurrence risk in Dupuytren's disease. J Hand Surgery Eur Vol 2013;38:424-429.

11. Noble J, Heathcote JG, Cohen H. Diabetes mellitus in the aetiology of Dupuytren's disease. J Bone Joint Surg Br 1984;66:322-325.

12. Thurston AJ. Dupuytren's disease. J Bone Joint Surg Br 2003;85:469-477.

13. Brown E, Genoway KA. Impact of diabetes on outcomes in hand surgery. J Hand Surg 2011:36:2067-2072.

14. Augoff K, Tabola R, Kula J, Gosk J, Rutowski R. Epidermal growth factor receptor (EGF-R) in Dupuytren's disease. J Hand Surg Br 2005;30:570-573. 
15. Ratajczak-Wielgomas K, Gosk J, Rabczyński J, et al. Expression of MMP-2, TIMP-2, TGF- $\beta$, and decorin in Dupuytren's contracture. Connect Tissue Res 2012; 53:469-477.

16. Carvalhana G, Auquit-Auckbur I, Milliez PY. [Dupuytren's disease: state of knowledge and research in physiopathology.] [Article in French]. Chir Main 2011;30:239-245.

17. Shih B, Tassabehji M, Watson JS, Bayat A. DNA copy number variations at chromosome $7 \mathrm{p} 14.1$ and chromosome $14 \mathrm{q} 11.2$ are associated with Dupuytren's disease: potential role for MMP and Wnt signaling pathway. Plast Reconstr Surg 2012;129:921-932.

18. Shih B, Watson S, Bayat A. Whole genome and global expression profiling of Dupuytren's disease: systematic review of current findings and future perspectives. Ann Rheum Dis 2012;71:1440-1447.

19. Wilkinson JM, Davidson RK, Swingler TE, et al. MMP-14 and MMP-2 are key metalloproteases in Dupuytren's disease fibroblast-mediated contraction. Biochim Biophys Acta 2012;1822:897-905.

20. Verhoekx JS, Beckett KS, Bisson MA, McGrouther DA, Grobbelaar AO, Mudera V. The mechanical environment in Dupuytren's contracture determines cell contractility and associated MMP-mediated matrix remodeling. J Orthop Res 2013;31:328-334.

21. Terek RM, Jiranek WA, Goldberg MJ, Wolfe HJ, Alman BA. The expression of platelet-derived growth-factor gene in Dupuytren contracture. J Bone Joint Surg Am 1995:77:19

22. Dolmans GH, Werker PM, Hennies HC, et al. Wnt signaling and Dupuytren's disease. N Eng J Med 2011;365:307-317.

23. McCarty, CA, Wilke, RA, Giampietro, PF, Wesbrook, SD, Caldwell MD. Marshfield Clinic personalized medicine research project (PMRP): design, methods, and recruitment for a large population-based biobank. Personalized Medicine 2005;2(1):49-79

24. Purcell S, Neale B, Todd-Brown K, et al. PLINK: a tool set for whole-genome association and population-based linkage analyses. Am J Hum Genet 2007;81:559-575.

25. Cross DS, Ivacic LC, Stefanski EL, McCarty CA. Population based allele frequencies of disease associated polymorphisms in the Personalized Medicine Research Project. BMC Genet 2010;11:51.

26. Degreef I, De Smet L, Sciot R, Cassiman JJ, Tejpar S. Betacatenin overexpression in Dupuytren'z disease is unrelated to disease recurrence. Clin Orthop Relat Res 2009;467:838-845.

27. Varallo VM, Gan BS, Seney S, Ross DC, Roth JH, Richards RS, McFarlane RM, Alman B, Howard JC. Beta-catenin expression in Dupuytren's disease: potential role for cellmatrix interactions in modulating beta-catenin levels in vivo and in vitro. Oncogene 2003;22:3680-3684.

28. O'Gorman DB, Wu Y. Seney S, Zhu RD, Gan BS. Wnt expression is not correlated with beta-catenin dysregulation in Dupuytren's disease. J Negat Results BioMed 2006;5:13.

29. Mosakhani N, Guled M, Lahti L, Borze I, Forsman M, Paakkonen V, Ryhanen J, Knuutila S. Unique microRNA profile in Dupuytren's contracture supports deregulation of $\beta$-catenin pathway. Mod Pathol 2010;23:1544-1552.

30. Colwell AS, Krummel T M, Longaker MT, Lorenz HP. Wnt-4 expression is increased in fibroblasts after TGF-betal stimulation and during fetal and postnatal wound repair. Plast. Reconstr. Surg 2006;117:2297-2301.

31. Akhmetshina A, Palumbo K, Dees C, et al. Activation of canonical Wnt signalling is required for TGF- $\beta$-mediated fibrosis. Nat Commun 2012;3:735.

32. Logan CY, Nusse R. The Wnt signaling pathway in development and disease. Annu Rev Cell Dev Biol 2004;20:781-810.
33. Verjee LS, Verhoekx JS, Chan JK, Krausgruber T, Nicolaidou V, Izadi D, Davidson D, Feldmann M, Midwood KS, Nanchahal J. Unraveling the signaling pathways promoting fibrosis in Dupuytren's disease reveals TNF as a therapeutic target. Proc Natl Acad Sci U S A 2013;110:E928-E937

34. Shih B, Tassabehji M, Watson JS, Bayat A. DNA copy number variations at chromosome $7 \mathrm{p} 14.1$ and chromosome $14 \mathrm{q} 11.2$ are associated with Dupuytren's disease: potential role for MMP and Wnt signaling pathway. Plast Reconstr Surg 2012;129:921-932.

\section{Author Affiliations}

Eric R. Anderson, DO ${ }^{*}$, , Zhan Ye, PhD ${ }^{\dagger}$, Michael D. Caldwell, MD, PhD , James K. Burmester, PhD ${ }^{\S, 2}$

"Surgical Resident, Marshfield Clinic, Marshfield, WI USA

'Biomedical Informatics Research Center, Marshfield Clinic

Research Foundation, Marshfield, Wisconsin, USA

${ }^{*}$ Department of General Surgery, Director of Wound Healing

Program, Marshfield Clinic, Marshfield, Wisconsin, USA

${ }^{\S}$ Clinical Research Center, Marshfield Clinic Research

Foundation, Marshfield, Wisconsin, USA

${ }^{I}$ Current Affiliation: Department of General Surgery, Divine Savior Health Care, Portage, Wisconsin, USA

${ }^{2}$ Current Affiliation: Division of Medical Research, Gundersen Medical Foundation, Gundersen Health System, La Crosse, Wisconsin, USA 